IS-038 OXIDATIVE STRESS RELATED DISEASES IN THE FETUS AND THE NEWBORN

G Buonocore, S Perrone. Department of Molecular and Developmental Medicine, University of Siena, Siena, Italy

\subsection{6/archdischild-2014-307384.38}

Many evidences have correlated neonatal diseases with oxidative stress (OS) caused by the harmful effect of free radicals (FRs). FRs are reactive oxygen and nitrogen species formed as a result of normal cellular metabolism and have many roles in cell signalling pathways. FRs can be also produced in the course of hypoxia, ischemia, ischemia-reperfusion, hyperoxia, inflammation and as consequence of exposition to many endogenous and exogenous oxidising agents. OS injury occurs when tissues, cells and biomolecules undergo an excessive exposition to oxidising agents, both endogenous (substances produced by inflammatory cells) and exogenous (environmental toxins). The FRs production exceeds antioxidant defences and OS occurs. OS is on the basis of several human pathologies such as stroke, hypertension, diabetes, rheumatic diseases, multiple sclerosis, neurodegenerative diseases and cancer. In Neonatology, OS is involved in the development of several FR-related diseases (FRRD) such as oxidative hemolysis, intraventricular haemorrhage, necrotizing enterocolitis, retinopathy of prematurity, chronic lung disease, renal failure. The damaging effect of FRs in perinatal period may be demonstrated by measuring OS biochemical markers in amniotic fluid and in cord blood. Intrauterine hypoxia induces OS in pregnancies with fetal growth restriction (FGR). Prostanoids concentration, actually considered as the best biomarker of OS, are particularly elevated in amniotic fluid of pregnancies with Down syndrome affected fetuses and in cord blood of newborns from maternal chorioamnionitis. They also have a significant predictive value to early detect pregnancies at high risk of premature rupture of membranes, fetuses suffering FGR and newborns who will develop the FRRD.

\section{IS-039 ANTIOXIDANT STRATEGIES: WHERE ARE WE NOW?}

F van Bel. Neonatology, University Medical Center Utrecht, Utrecht, Netherlands

\subsection{6/archdischild-2014-307384.39}

Part of asphyxia-related brain damage occurs upon reoxygenation. Renewed availability of oxygen activate biochemical pathways and neuronal cell death. Important pathways are: 1) Calcium-induced formation of neurotransmitters; 2) formation of (pro-) radicals; 3) activation of inflammation; 4) induction of apoptosis; 5) depletion of growth factors. Four important sources of free radicals are: 1) Nitric oxide (NO)related formation of peroxynitrite. It is reported that selective iNOS/eNOS inhibitor 2-iminobiotin induced neuroprotection after asfyxia in animal models. 2) Pro-radicals, such as non proteinbound-iron (NPBI), lead to formation of hydroxyl free radicals. NPBI chelation with deferoxamine, which has also a stabilising effect on HIF1-alpha and stimulates trophic factors, showed encouraging results in experimental models. 3) Formation of superoxide radical by metabolisation by xanthine-oxidase (XO) can be blocked by XO-inhibitors such as allopurinol. 4) Metabolisation of arachidonic acid to prostaglandin leading to superoxide can be blocked by cyclo-oxygenase inhibitors. Since XO-derived superoxide occurs upon reoxygenation after asphyxia, a trial with allopurinol to the mother with signs of perinatal fetal hypoxia has been started. Activation of inflammatory factors after asphyxia is recognised to be related to post-aphyxial brain damage.

Rather than monotherapy directed to one pathway, a combination of drugs intervening in various pathways in relation with the time-profile of these pathways, might achieve optimal reduction of reperfusion injury.

\section{Long-term Outcome After Critical Illness: The Need for Appropriate Care Continues After Discharge from the ICU}

\section{IS-040 CRITICAL ILLNESS: EARLY RECOGNITION OF STRESS AND ITS LONG-TERM IMPACT ON THE CHILD AND FAMILY IS IMPORTANT TO OFFER THE RIGHT INTERVENTIONS}

M Grootenhuis. Psychosocial, Emma Childrens Hospital, Amsterdam, Netherlands

\subsection{6/archdischild-2014-307384.40}

Children and their parents who are exposed to medical lifethreat due to illness or injury are at risk for developing symptoms of posttraumatic stress of psychosocial problems which should be monitored during follow up and treated. Several initiatives which can be used during follow-up using the internet are presented.

A set of material that could be used is the Paediatric Traumatic Stress Toolkit (http://www.nctsnet.org/). This toolkit is created by the National Child Traumatic Stress Network (NCTSN) and comprises both practical tools and training materials to health care professionals to enhance trauma-informed practice. After considering the A-B-C's (airway-breathing-circulation), professionals should consider the D-E-F's (distress-emotional support-family) we modified the material for Dutch families and health care professionals www.nahetziekenhuis.nl and evaluated this material.

Health Related Quality of Life (HRQOL) questionnaires are increasingly used in clinical practice. These Patient Reported Outcomes (PROs) are provided to the paediatrician to facilitate communication with patients during a consultation. Currently, the use of PROs in daily clinical practice is very time consuming and often has logistical problems. The use of a web-based programme can overcome these problems and contributes to an improved use of PROs in clinical practice. We therefore developed an easily accessible website (www.hetklikt.nu) for outpatient treatment and a training programme for paediatricians to maximise the effectiveness and the practical use of PROs (for demonstration: username PRO and password: KLIK).

\section{Neonatal Extracorporeal Membrane Oxygenation, Risk Factors for the Brain and Long Term Neurological Outcome}

\section{IS-041 NEONATAL ECMO AND RISK FACTORS FOR THE BRAIN}

AC de Mol. Pediatrics - Neonatology, Albert Schweitzer Hospital, Dordrecht, Netherlands

10.1136/archdischild-2014-307384.41

Background Neonatal extracorporeal membrane oxygenation (ECMO) is a lifesaving therapeutic approach in newborns suffering from severe, but potentially reversible, severe respiratory 
insufficiency, mostly complicated by persistent pulmonary hypertension. However, cerebral damage, intracerebral haemorrhage as well as ischemia belong to the most devastating complications of ECMO.

Objectives Objectives of the presentation are to give insights into what is known from literature concerning cerebral damage related to neonatal ECMO treatment for pulmonary reasons.

Methods A short introduction in ECMO indications and technical aspects of ECMO are provided for better understanding of the process. Against the results of the only multicentre randomised trial of ECMO versus conservative treatment, the presentation will focus on (potential) risk factors for cerebral haemorrhage and ischemia during ECMO treatment.

Results and conclusion Although neonatal ECMO treatment shows improved outcome compared to conservative treatment in cases of severe respiratory insufficiency, it is related to disturbances in various aspects of neurodevelopmental outcome. Risk factors for cerebral damage are either related to the patient's disease, ECMO treatment itself, or a combination of both.

It is of on-going importance to further understand pathophysiological mechanisms resulting in cerebral haemorrhage and ischemia due to ECMO and to develop neuroprotective strategies and approaches.

\section{IS-042 NEONATAL EXTRACORPOREAL MEMBRANE OXYGENATION, NEUROIMAGING AND OUTCOME}

${ }^{1} \mathrm{~A}$ van Heijst, ${ }^{2} \mathrm{AC}$ de Mol, ${ }^{3} \mathrm{H}$ IJsselstijn. ${ }^{1}$ Neonatology, Radboud University Nijmegen Medical Centre, Nijmegen, Netherlands; ${ }^{2}$ Pediatrics, Albert Sweitzer Hospital, Dordrecht, Netherlands; ${ }^{3}$ Pediatric Surgery and Pediatric Intensive Care, Erasmus MC-Sophia Children's Hospital, Rotterdam, Netherlands

10.1136/archdischild-2014-307384.42

\begin{tabular}{|c|c|}
\hline Abnormal finding & Score \\
\hline \multicolumn{2}{|l|}{ Ventricular Dilatation $(r w=1)$} \\
\hline Minimal & 1.0 \\
\hline Moderate & 2.0 \\
\hline Marked & 3.0 \\
\hline \multicolumn{2}{|l|}{ Subarachnoid Space $(r w=1)$} \\
\hline Wide interhemsipheric fissure & 0.5 \\
\hline Large subarchnoid space & 1.0 \\
\hline \multicolumn{2}{|l|}{ Haemorrhage $(r w=2)$} \\
\hline Subependymal only & 0.5 \\
\hline Single petechial & 0.5 \\
\hline Scattered petechial & 1.0 \\
\hline Intraventricular & 1.0 \\
\hline Small parenchymal $(<1 \mathrm{~cm})$ & 1.5 \\
\hline Large parenchymal & 3.0 \\
\hline Extraaxial small & 0.5 \\
\hline Extraaxial large & 1.0 \\
\hline \multicolumn{2}{|l|}{ Parenchymal lesions $(r w=3)$} \\
\hline Focal PVL or hypodensity & 0.5 \\
\hline Focal atrophy & 0.5 \\
\hline Patchy PVOL of hypodensity & 2.0 \\
\hline Diffuse PVL or hypodensity & 3.0 \\
\hline Mild generalised atrophy & 2.0 \\
\hline Moderate generalised atrophy & 3.0 \\
\hline Mass lesion/infaction & 3.0 \\
\hline
\end{tabular}

ECMO in neonates: neuroimaging findings and outcome Extracorporeal membrane oxygenation (ECMO) is a rescue therapy for newborns with severe but reversible respiratory failure. Although ECMO has significantly improved survival, it is associated with substantial complications of which intracranial injuries are the most important. These injuries consist of haemorrhagic and nonhaemorrhagic, ischaemic lesions. Different from the classical presentation of haemorrhages in preterm infants, in ECMO treated newborns haemorrhages are mainly parenchymal and with a high percentage in the posterior fossa area. There are conflicting data on the predominant occurrence of cerebral lesions in the right hemisphere. The existence of intracerebral injuries and the classification of its severity are the major predictors of neurodevelopmental outcome. This section will discuss the known data on intracranial injury in the ECMO population and the effect of ECMO on the brain.

\section{Rare Diseases, Common in Paediatrics}

\section{IS-043 COMPREHENSIVE COORDINATED CARE FOR CHILDREN WITH RARE CONDITIONS}

C Cooley. Pediatrics, Crotched Mountain Foundation, Greenfield NH, USA

\subsection{6/archdischild-2014-307384.43}

Most of the chronic conditions of childhood occur relatively rarely, and many of those rare conditions require complex care. Children with the most complex conditions comprise 5\% or less of the paediatric population but account for as much as $70 \%$ of paediatric care expenditures. These children are particularly vulnerable to the effects of fragmented care and services resulting in less than optimal health outcomes and higher health care costs. Developed by paediatricians, the medical home model has been promoted by the United States Maternal and Child Health Bureau and the American Academy of Paediatrics as a locus of proactive, coordinated care in the context of an integrated system of child health services and supports. The medical home has now been adopted as a model of care across the life span and occupies a critical position in United States health care reform efforts. This presentation provides an overview of the medical home model and its place in an integrated care model of child health for children with rare and complex conditions. The critical functions of care coordination, written and shared care plans, and explicitly articulated co-management roles for primary care providers, specialists, other ancillary service providers, and families will be explained.

\section{IS-044 APPRAISAL OF DISABILITY IN RARE DISEASES WITH THE ICF-CY: THE ORPHANET DISABILITY PROJECT}

M de Chalendar, S Bee, A Olry, A Rath. Inserm-US14, Orphanet, Paris, France

\subsection{6/archdischild-2014-307384.44}

Aim Very little information is available about the disabilities encountered by rare disease (RD) patients, of whom more than half are children. The Orphanet (www.orpha.net) Disability Project aims to improve the knowledge and visibility of disabilities associated with RDs.

Method We are indexing the functional consequences of each RD with the Orphanet Functioning Thesaurus, an adaptation from the "Activities and participation" and "Environmental 